\author{
MIESZEK JAGIEŁŁO \\ Uniwersytet im. Adama Mickiewicza, Poznań
}

\title{
DIE THEBANISCHE SINTFLUTSAGE
}

ABSTRACT: The Ogygian Flood is one of the lesser known flood myths. Unfortunately, there are only late and very vage accounts of it. Certain passages from Homer and Hesiod however allow a tentative reconstruction of the Theban deluge. Furthermore, a comparatistic approach gives the opportunity to adduce earlier, more elaborate oriental flood myths in order to get a glimpse of some of the facettes of the Theban adaptation of these myths. The paper includes some remarks on the

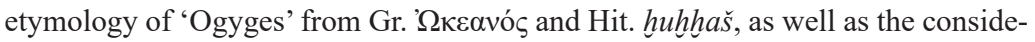
ration that the demon Pašittu (Lamaštu) is in fact the Theban sphinx and therefore, Hera should be identified with Nintu.

KEY WORDS: flood, deluge, Thebes, Ogyges, Sphinx, Lamaštu, Pašittu, Nintu

\section{EINLEITUNG}

Es ist ein wiederkehrendes Motiv - die Flut, die der Menschheit ein Ende setzen soll, kommt als Strafe der kompromisslosen Götter. Nur ein Bruchteil kann überleben, um einen Neubeginn in einer neuen, gereinigten Werte- und Weltordnung zu wagen. Ungefähr so stellt sich das Motiv dar. Es begegnet uns in allerlei mediterranen Zivilisationen: vom Zweistromland bis in die Ägäis. Als wohl erste Überlieferung einer solchen Flut gilt das akkadische Atrahasis-Epos, etwa 1600 v. Chr. in akkadischer Sprache auf drei Tafeln verewigt. Dessen Spuren finden sich auf der vorletzten, elften Tafel des babylonischen GilgameschEpos. Der modernen Welt wurde die Geschichte des Utnapischtim, des 
babylonischen Gegenstücks zu Atrahasis, erst im Jahre 1853 zugänglich, als das Archiv des Palastes zu Niniveh, die sog. Bibliothek des Assurbanipal, von den Archäologen um Sir Austin Henry Layard freigelegt wurde. Eine Version des Mythos, die Berossos in seinem auf Griechisch verfassten Werk Babyloniaka überlieferte, ist auf einer verdächtig spät entstandenen Tafel mit einer sumerischen Inschrift (wahrscheinlich einer zu liturgischen Zwecken angefertigten Übersetzung aus dem Akkadi-

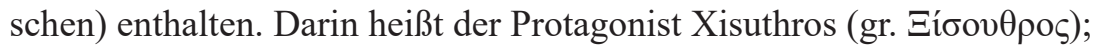
die sog. sumerische Köngisliste nennt ihn Ziusudra ${ }^{1}$. Einer dieser mesopotamischen Versionen folgt die biblische Erzählung von Noah (hebr. נָֹ in Gen. 1: 6, 5-9, 28 und dessen koranischer Variante Nuh (arab. نُوحُ) hauptsächlich in Sura $71 A n-N u h$, teilweise auch in Sura 11 Hud (V. 37). In diese Tradition fügt sich auch der griechische Mythos um Deukalion und Phyrra ein (Pind. O. IX 43-55; Hellanicus FGrH 4 F 117; Epicharm fr. 113, 4-15; Plat. Tim. 22a; Apollodor. I 7, 2; Hyginus Fab. 153; Ov. Met. VII 353-355; Diod. XIV113, 2; Strab. VII 7, 2).

Aber auch andere Mythen berichten den Untergang einer Zivilisation durch eine Flutkatastrophe, so zum Beispiel jene der Platonischen Atlanter (Tim. 25a-d; Criti. 113c-121d) oder jene der Telchinen (Diod. V 55, 1-56, 2). Die Metamorphosen des Ovid erzählen zweierlei Flutmythen. Die Flut des Deukalion ist - wie bereits erwähnt - eine Adaption des mesopotamischen Mythos. Die Sage des Philemon und dessen Gemahlin Baucis ist dagegen eine Ätiologie des Tempels zu Ehren Zeus' und Hermes' (Ov. Met. VIII 611-724). William M. Calder sieht hier eine anatolische Version des Motivs, deren Herkunft er in den Gebieten um Apamea und Iconium sucht. Unter anderem sei eine Besonderheit der anatolischen Fassung die Überschwemmung quasi aus dem Grundwasser heraus: Die Flut ströme aus dem Erdboden, anstatt die Welt durch Regengüsse zu überfluten².

Lambert 1969: 1-25, 138-145.

Calder 1988: 101-111. 


\section{DIE SINTFLUT DES OGYGES}

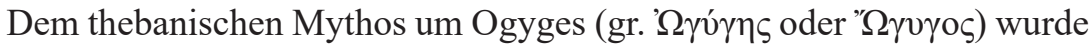
bisher weniger Beachtung geschenkt. Robert Louis Fowler hatte die bis dato zugänglichen Quellen der Sage untersucht. Doch sie sind sehr mager: Die erste Erwähnung findet sich - obwohl umstritten - im 6./5. Jh.

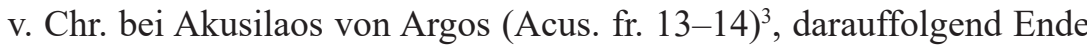
des 5. Jh. v. Chr. bei Hellanikos von Lesbos (Hellanic. fr. 62) ${ }^{4}$ und um das Jahr 37 v. Chr. unanfechtbar bei Varro (Res Rust. III 1, 2-4):

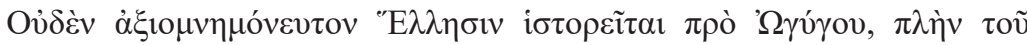

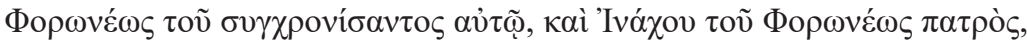

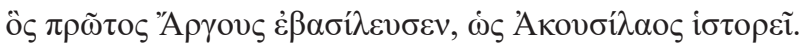

Nichts von Belangen wurde von den Hellenen überliefert (von der Zeit) bevor Ogygos, außer dass Phoroneos dessen Zeitgenosse war, und (auch) Inachos, des Phoroneos Vater, der als Erster die Argolis beherrschte - so berichtet Akusilaos. ${ }^{5}$

Acus. fr. 13

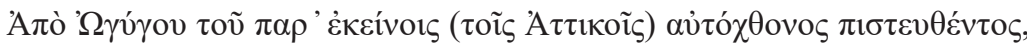

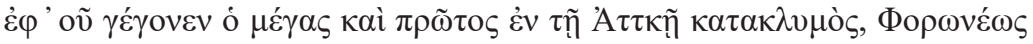

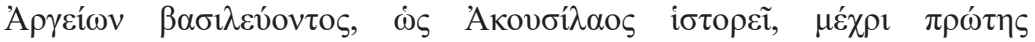

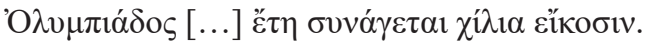

Seit Ogygos, vom dem man glaubte, dass er auch (zusammen mit den Attikern) autochton sei, bevor nicht die große und erste Sintflut in der Attis eintrat, herrschte Phoroneos der Argiver - so berichtet Akusilaos bis zur ersten Olympiade [...] insgesamt eintausendundzwanzig Jahre [vergingen]. ${ }^{6}$

Acus. fr. 14

Vgl. DFHG. Zu Akusilaos siehe auch die Bearbeitung von Robert L. Fowler (2007: 1-28).

$4 \quad$ Vgl. DFHG, s. auch hier Fowler (2007: 147-231).

5 Übers. M.J.

6 Übers. M.J. 


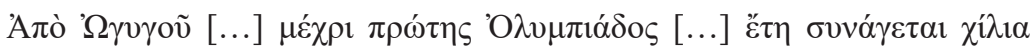

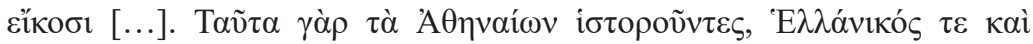

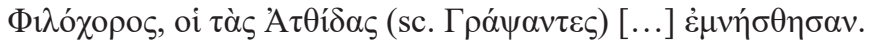

Seit Ogygos [...] bis zur ersten Olympiade [...] insgesamt eintausendundzwanzig Jahre [vergingen] [...] Derartiges erzählten sie (?) sowohl den Athenern, als auch Hellanikos und Philochoros, der Atthiden " (Verfasser) [...] erinnert wurden.?

Hellanic. fr. 62

Etenim vetustissimum oppidum cum sit traditum Graecum Boeotiae Thebae, quod rex Ogyges aedificarit [...] Thebae, quae ante cataclysmon Ogygi conditae dicuntur, eae tamen circiter duo milia annorum et centum sunt.

Ist ja doch als älteste griechische Stadt Boiotiens Theben überliefert, das König Ogygos erbaute [...] Theben, das vor der Sintflut des Ogyges gegründet worden sein soll, es ist ungefähr zweitausendeinhundert Jahre alt. ${ }^{8}$

Varro, Res Rust. III 1, 2-3

Zudem sieht es so aus, als würden Julius Africanus (Afric. fr. 22) ${ }^{9}$ und Pseudo-Justin (Cohortatio ad Graecos IX 2) ${ }^{10}$ zwar die Ogygische Sintflut bei Akusiloas und Hellanikos bestätigen, dabei jedoch Ogyges

7 Übers. M.J.

8 Übers. nach D. Flach.

9 Darauf verweist Robert L. Fowler (siehe Fußnote 14). Hierbei handelt es sich um Afric. Chron. XII, 2 [1076]. Nach meinem Dafürhalten verwirft Julius Africanus, der durch Philochoros beinflusst wurde, die Idee eines König Ogyges und dessen Sintflut als Ganzes, wie im anschließenden Afric. Chron. XIII, 1 deutlich wird. Überhaupt kennt er wohl eigentlich nur eine attische Ogyges-Überlieferung. In Bezug auf Julius Africanus schreibt Umberto Roberto in seiner Zusammenfassung von From Hellenistic to Christian Universal History. Julius Africanus and the Atthidographers on the Origins of Athens: ,Interessant ist, wie Africanus die Autochtonie und kulturelle Eigenständigkeit Athens ablehnt. Die Polemik gegen eine idealisierende Beschreibung der Urgeschichte Athens ist eine wichtige Etappe im Wandel von einer klassisch-hellenistischen zu einer christlichen Universalgeschichte“ (2011: 525-539).

10 Leider ist es mir gelungen, lediglich auf diese Quellen in der englischen Übersetzung von Thomas B. Falls (2008: 367-424) zurückzugreifen. Pseudo-Justin ist aber keinesfalls in seiner Einschätzung der Historizität Ogyges' so radikal wie Julius Africanus. Einige glauben jedoch, Africanus habe Justin gefolgt (vgl. Staab 2006: 66) (Fußnote 27). 
als König der Attik negieren, vielleicht aber gleichzeitig dessen Herrschaft über Theben attestieren. Dazu kommen noch Panyassis ${ }^{11}$ (fr. 24 zitiert in Hek. fr. 10) ${ }^{12}$ aus dem 5. Jh. v. Chr. und die nur schwer (wohl auf die Zeitspanne zwischen dem 6. und 4. Jh. v. Chr.) zu datierende Korinna (PMG fr. 671) ${ }^{13}$, die Ogyges auch erwähnen (Fowler 2013: $118-120)^{14}$.

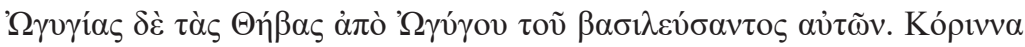

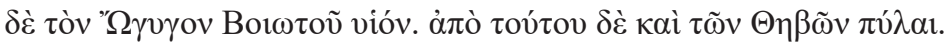

Theben wird Ogygia (d.h. Ogygisch) genannt, nach Ogygos, der darüber herrschte. Korinna weiß Ogygos als des Böotos Sohn. Nach ihm sind auch die Tore Thebens benannt. ${ }^{15}$

Korinna PMG fr. 671

Doch schon in der Epik tauchten Derivate des Namens Ogyges immer mit aquatischen Konnotationen auf. Kirkes homerische Insel Ogygia (gr. ' $\Omega \gamma v \gamma i \alpha$ ) - eine klare Anspielung auf Dilmun, den Wohnort des Utnapischtim ${ }^{16}$ - wird in der Odyssee (Od. I 85; VI 172; VII 244; VII 254; XII

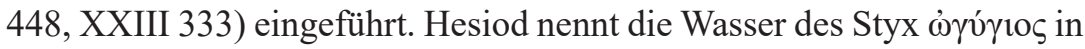
Hes. Th. 805-806. Ogyges selbst wird ohne Bezug auf die Flut als Herrscher des böotischen Thebens von Pausanias (Paus. IX 5, 1) genannt.

Hinsichtlich dessen Person bestehen zahlreiche Hypothesen. Zum

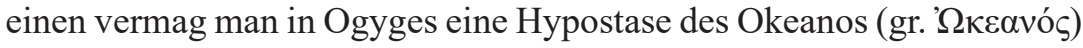
zu erblicken. Laut Paulys Realencyclopädie der Classischen Altertumswissenschaft wiese die Etymologie auf einen Ursprungsort in Karien ${ }^{17}$ hin. Bereits 1819 zählte Philipp Karl Buttmann in einem seiner Aufsätze

\footnotetext{
$11 \quad$ Vgl. Matthews 1976: 100-106.

12 Leider habe ich das betreffende Fragment nicht selbst in Augenschein nehmen können.

13 Campbell 1992: 48-49.

14 Fowler 2013: 118-120.

15 Übers. M.J.

16 Kozłowski 2018: 11-31.

17 Wissowa 1937: 2076-2078, s.v. „Ogygos“. Anhand der mythischen Genealogie, die Panyassis überliefert, argumentieren Takmer und Akdodu-Arca (2001/2002: 1-34) in einem Artikel, Ogyges sei in der Tat Karier gewesen.
} 
Argumente auf, die beide Figuren etymologisch zu verbinden ${ }^{18}$; „Ogen und Ogenos war für den Namen Okeanos eine ältere Form, die nur bei einigen alten oder gelehrten Schriftstellern gefunden wird", schrieb er in Bezug auf Hesychios und Lykophron. Durch eine Reduplikation des Stammes, könne man „Ogyges“ aus „Okeanos“ ableiten. Hugo Ehrlich stimmte dem zu, doch elaborierte eine Bedeutungsverschiebung in Richtung ,unterweltlich“19. Richard S. Cadwell tendiert eher zu der Bedeu-

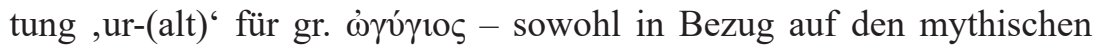

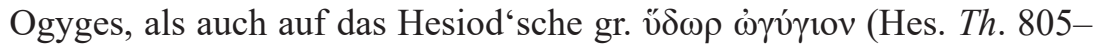
$806)^{20}$. Darin folgte er wohl Hesiods Scholiasten, der erklärt - wie Buttmann zitierte - „Ogygisch heiße was alt ist, von Ogyges, dem ehemaligen Könige aller Götter"'21. Zum anderen wird auf eine mögliche Verbindung von Ogyges zum biblischen Og von Basan (hebr. עוג, gr. $\Omega \gamma$ ) verwiesen. Og war ein Refaiter (hebr. רפָָָּּ, phön. rp 'm) 22 (Jos. 12: 4; Deut 3:11), der - laut der Midrasch-Tradition - Noah beim Bau der Arche (hebr. ${ }^{23}$, gr. $\lambda \alpha{ }^{2} \pi \nu \alpha \xi$, lat. arca) assistierte und von Noah zum Dank dafür geborgen wurde ${ }^{24}$. Das byzantinische Biblion Suda gibt unter dem Ein-

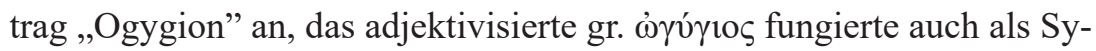

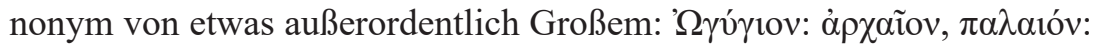

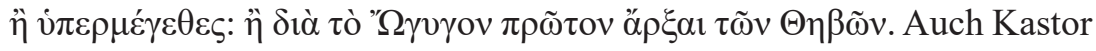
von Rhodos, der Ogyges einen der Titanen nennt (FGrHist 250 F9, vgl.

18 Buttmann 1828/1829: 205-208. Zuerst veröffentlicht in Buttmann 1819: 48-54.

19 Ehrlich 1908: 636-639.

20 Cadwell 1987: 37.

21 Buttmann 1928/1929: 207. In einer Fußnote führte er zudem an, es sei aus ảंò

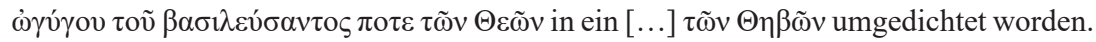
22 Interessanterweise wird das Hebräische in der Septuaginta verschiedenartig über-

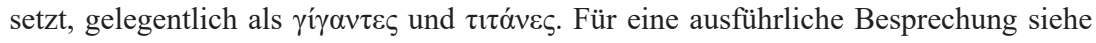
Niesiołowski-Spanò 2015: 73-76.

23 John Pairman Brown (1995: 35, Fußnote 135) gibt an, dass das böotische Toponym Thebe (myk. te-qa) mit hebr. תבָָ (tevah), der biblischen Bezeichnung für die Arche Noahs, assoziiert worden sei. Er kann dafür keine semantische Erklärung finden. Durch den hier beschriebenen thebanischen Sintflutmythos sei eine solche semantische Verbindung gefunden, nichtsdestotrotz bleibe ich dieser Etymologie gegenüber skeptisch.

$24 \quad$ Vgl. Friedländer 1916: 167. Darüber hinaus attestieren zwei Texte, der eine Ugaritisch (KTU 1. 108=RS 24.252; aus dem 13. Jh. v. Chr., siehe Wyatt 2006: 395-398), der andere Phönizisch (aus dem 5. Jh. v. Chr., Rölling 1974: 1-15), einen Kult des Og im Levant. 
auch FGrHist 250 F1), könnte darauf anspielen. Für Scott B. Noegel ist die Ähnlichkeit der Figuren Ogyges und Og triftig, besonders hinsichtlich der jüdischen Überlieferungen nach der Zeitenwende ${ }^{25}$. Doch Martin L. West postulierte in Bezug auf eine Ogygische Sintflut, Flutmythen hätten Griechenland nicht vor dem Jahr 550 v. Chr. erreicht ${ }^{26}$.

Die griechische Mythologie kennt des Weiteren drei (titanische) He-

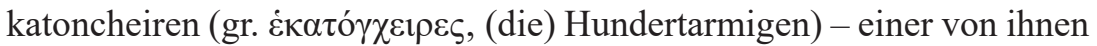
heißt laut Hesiod (Th. 419; 618; 714; 734; 817) Gyes (gr. Гúnऽ), anderen

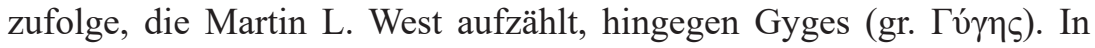
Hes. Th. 815-819 wird berichtet, die Hekatoncheiren lebten ,in Wohnungen dort an Okeanos" untersten Gründen" ${ }^{\text {“6 } 7}$ und stünden in besonderer Verbindung mit Poseidon ${ }^{28}$. Etymologisch zieht West die Form Gyges dem Hesiod'schen Gyes vor und vermutet nach Friedrich Gottlieb Welcker, diese habe sich aufgrund einer Assoziation an das gr. yoĩov für ,Glied, Hand', angepasst ${ }^{29}$. Mary R. Bachvarova erörterte (ohne auf Ogyges hinzuweisen) die Namensgebung im Westanatolien der Bronzezeit bis ins 7. Jh. v. Chr. Allem Anschein nach dienten die Namen mit dem hethitischen Element heth. huhha- ,Großvater, Ahne' als Werkzeug dynastischer Legitimierung, darunter auch der Name Gugēs (Gyges). Demnach etablierte sich heth. huhhaš als mythischer Vorvater in der Namensgebung der Herrscherkasten, auch über das Hethiterimperium hinaus $^{30}$. Außerdem entstanden auch Monumente zu Ehren von Gyges. Im

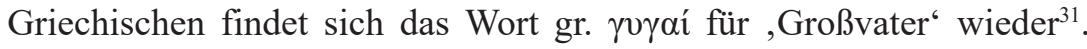
Noah Webster hatte bereits 1810 darauf hingewiesen, dass Gyges von den Griechen wohl mit Ogyges assoziiert wurde, obwohl er keine gemeinsame Etymologie der beiden Namen vermutete ${ }^{32}$. Auch Joseph E.

\footnotetext{
$25 \quad$ Noegel 1998: 411-426.

26 West 1997: 489-493.

27 Übers. nach J.H. Voß.

28 Sowa 1984: 152.

29 West 1966: 210.

30 Bachvarova 2016: 375-376.

31 Bachvarova 2016: 375. Wichtig sei auch, dass „die Form Gugēs, mit initialem Velar" karischen Ursprungs ist. Folglich führten sowohl die etymologische Hypothese

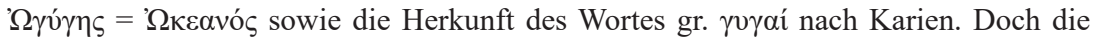
hier bisher nur vage angedeutete Verbindung von Ogyges und Gyges ist natürlich nicht selbstverständlich.

32 Webster 1810: 199-200, s.v. „Ogyges”.
} 
Fontenrose verwies, ganz im Einklang mit Buttmann und Ehrlich, auf eine Verbindung aller möglichen Varianten - Ogyges, Gyges, Gog und Og, Ogen, Okeanos - als einander entsprechende grammatikalische Reduplikationen. Seine Vermutung geht jedoch weiter. Er setzt Ogyges - als den zuvor genannten Titanen und $\beta \alpha \sigma 1 \lambda \varepsilon v ́ \sigma \alpha \nu \tau o \zeta \pi o \tau \varepsilon \tau \tilde{\omega} \nu \theta \varepsilon \tilde{\omega} \nu-$ den Urgestalten Ophion (dem sog. Geron, dem Alten Mann), Okeanos, sowie Chaos gleich ${ }^{33}$. Dazu verweist er auch auf Einträge von Hesychios:

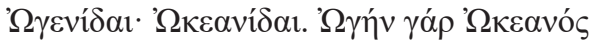

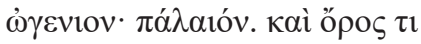

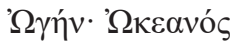

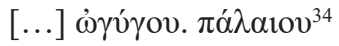 \\ Hsch. $\omega$ 19-27
}

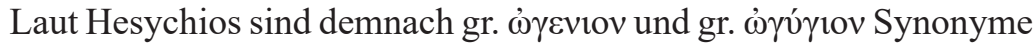

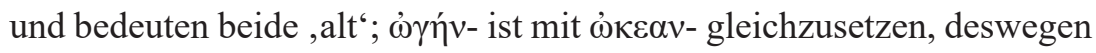
auch $\omega \gamma \gamma$ $\gamma-$. Dies sind jedoch keinesfalls etymologische, sondern eher

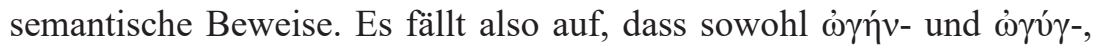
als auch die griechischen Ableitungen von heth. huhha- nicht nur eine gewisse phonetische, sondern auch eine semantische Ähnlichkeit aufzeigen $^{35}$. Dennoch bleibt es ein Rätsel, weshalb und wie es dazu gekommen sei, dass Og von Basan, eine karische (aquatische?) Ahnenfigur im wohl weitaus älteren biblischen Flutmythos auftrete ${ }^{36}$. Es ist aber klar, dass die heutige Wissenschaft mit den ihr zur Verfügung stehenden Mitteln und Quelltexten weder die Form ' $\Omega \gamma v ́ \gamma \eta \varsigma$ mit initialem Omega von „Gyges“ abzuleiten weiß, noch eine Reduplikation als Ursache für die Wandlung von „Okeanos“ zu „Ogyges“ als vertretbar gelten lassen kann.

\footnotetext{
$33 \quad$ Fontenrose 1974: 237 (Fußnote 27).

34 Latte, Hansen, Cunningham 2009: 256; ggf. auch die ältere Fassung: Schmidt 1867: 1578 .

35 Somit ergibt sich ein Verdacht, dass sowohl Homer, als auch Hesiod auf eine gewisse Art und Weise - direkt oder indirekt - aus dem Karischen (' $\Omega \gamma v ́ \gamma \eta \varsigma$ und „die Form Gugēs, mit initialem Velar") schöpfen konnten. In Karien belegt Homer auch einen Gygischen See (Il. II 865). Webster (1810: 200) verweist ebenfalls auf eine karische Gottheit mit dem Namen Ogoa.

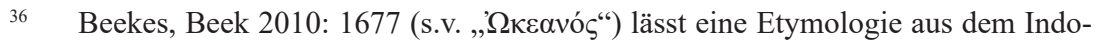
europäischen überhaupt nicht zu.
} 


\section{DIE THEBANISCHE SINTFLUT IN DER ILIAS}

Daniel W. Berman argumentierte, dass die fiktionalisierte, mythische Topografie der Stadt Ilios, samt Stadtmauern und Umgebung, in der Ilias mit denselben Worten beschrieben wurde, wie die Stadt Theben in der Homerischen und Hesiod'schen Epik. So sei das epische Troja aus einer Analogie zu einem epischen Theben heraus entstanden. Daraus zieht er den Schluss, dass die Inspiration für die homerischen Verse aus einem anderen, früheren Epos stammte - einem Epos, der der Stadt Theben, deren Mauer und den Geschehnissen vor den Stadttoren gewidmet war ${ }^{37}$, mit Wahrscheinlichkeit einem epischen Sieben gegen Theben. Schon in der Antike untersuchte man die Passagen Hom. Il. VII 336-354 und VII 436-441, die den Bau eines Verteidigungswalles um das Lager der Achaier herum beschreiben. Die Achaier bauten darin Zugangstore ein und bemannten diese. Auf eine recht simple Art und Weise bewies schließlich Henk W. Singor, dass es genau sieben dieser Tore gab ${ }^{38}$. Folglich stammte diese Episode aus einem früheren Epos, der einer Stadt gewidmet war, die über dieselbe Anzahl an Toren verfügte. Die Schlussfolgerung ist eindeutig: die homerischen Verse waren ursprünglich für

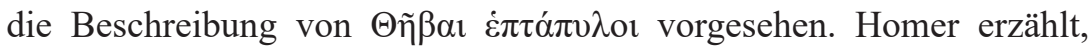
dass Poseidon für den Bau dieser Mauer von den Achaiern eine Hekatombe erwartete; die Götter erhielten auch ein angemessenes Opfer. Die Achaier hatten eine so gewaltige Mauer errichtet, dass diese sogar jene von Ilios und somit das Werk der Götter Apollo und Poseidon, die jene trojanische Befestigung gebaut hatten, übertraf. Die Götter beschlossen die Hybris der Achaier erst dann zu bestrafen, als die Mauern Trojas gefallen waren. Zeus lies es regnen, Poseidon sandte Ströme von Wasser in das Lager und lies es ins Meer spülen.

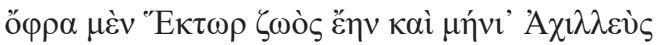

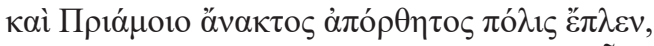

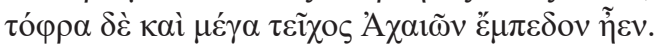

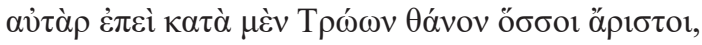

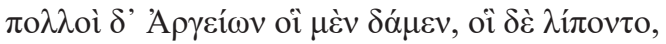

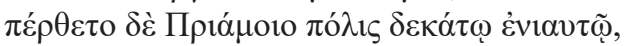

37 Berman 2015: 2-48.

38 Singor 1992: 403. 


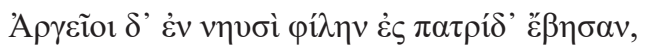

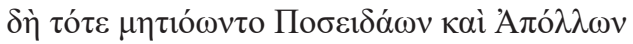

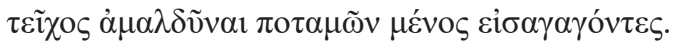

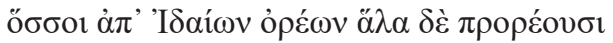

Hom. Il. XII 10-19

Denn weil Hektor lebend noch war, noch zürnet' Achilleus, und unzerrüttet die Stadt des herrschenden Priamos ragte; eben so lang' auch bestand der Danaer mächtige Mauer, aber nachdem gestorben der Troer tapferste Helden, mancher auch der Argeier vertilgt war, mancher noch übrig, und nun Priamos Stadt hinsank im zehnten der Jahre, dann die Argeier in Schiffen zur Heimat wiedergekehret; jetzo beschloss Poseidon im Rat und Phoibos Apollon, wegzutilgen den Bau, der Ströme Gewalt hinlenkend. Alle die hoch vom Idagebirg' in das Meer sich ergießen... ${ }^{39}$

\section{Und weiter:}

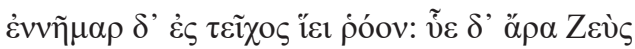

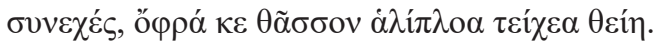

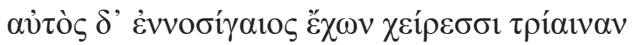

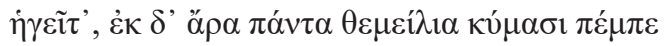

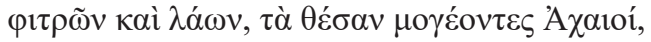

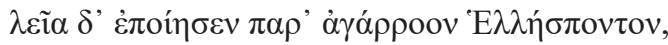

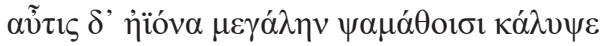

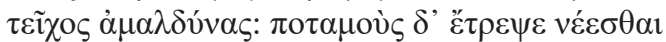

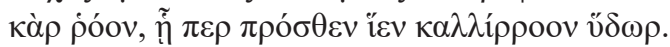
Hom. Il. XII 25-33

Gegen den Bau; neun Tage beströmt` er ihn; während herab Zeus regnete, schneller ins Meer die umflutete Mauer zu wälzen. Aber der Erderschütterer selbst, in den Händen den Dreizack, ging voran, und stürzt' aus dem Grunde gewühlt in die Wogen alle Blöck' und Steine, die mühsam gelegt die Achaier; schleift‘ und ebnet' es rings am reißenden Hellespontos, und umhüllte mit Sand weithin das große Gestade, wo er die Mauer vertilgt; dann wandt' er zurück in das Flutbett... ${ }^{40}$

39 Übers. nach J.H. Voß.

40 Übers. nach J.H. Voß. 
Singor zufolge gleicht die Hybris der Achaier jener der Erbauer des Turmes zu Babel ${ }^{41}$. In seiner Hypothese der ursprünglichen, siebentorigen Mauer Thebens, argumentiert Singor, die Thebaner haben es gewagt, die Befestigung des Palastes im Totenreich nachzubauen ${ }^{42}$. Die mesopotamischen Mythen berichten, dass Ganzer - der mesopotamische Unterweltpalast - habe sieben Mauerringe mit jeweils einem Tor gehabt. Jene sollten von den Thebanern nachgebaut worden und Ursprung des Zornes der Götter gewesen sein. Später, zu der Zeit als der Mythos um die Sieben gegen Theben entstand, hatte man die Konstruktion zu einem Mauerring mit sieben Toren umgedichtet und vereinfacht. Weshalb bloß? Nun, in den mesopotamischen Mythen, die vom Irkalla erzählen (z.B. Innanas Gang in die Unterwelt oder Nergal und Ereškigal), spielen die sieben Tore eine weitaus wichtigere Rolle als die sieben Mauern. Ištar zieht beispielweise ein Schmuck- oder Kleidungsstück an jedem der Tore aus, ehe sie hindurchschreiten darf (Innanas.., Z. 114-163). AuBerdem werden die Tore - nicht die Mauern - von Dämonen bewacht: jedes Tor von einem Dämon (jeweils Nedu, Enkišar, Endašurimma, Enuralla, Endukuga, Endušuba oder Ennugigi) ${ }^{43}$, nach dem auch die jeweilige Pforte benannt ist. Genauso hüten auch jeweils ein Verbündeter des Eteokles eine der sieben thebanischen Pforten im Mythos um die Sieben gegen Theben. Ehrlich meinte, dass die ' $\Omega \gamma v ́ \gamma 1 \alpha \iota \pi v \lambda_{\alpha} \alpha_{1}$ aus Eurip.

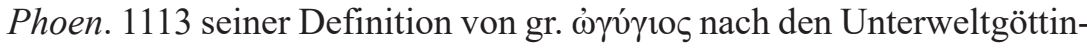

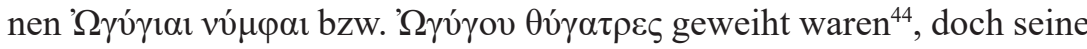
Argumentation stützt sich auf den Annahmen, es habe keine thebanische Sintflutüberlieferung gegeben und Ogyges habe ursprünglich nichts mit Theben gemeinsam gehabt.

Die Ströme, die von Poseidon und Apollo zum Überschwemmen des achaischen Lagers mobilisiert werden, sind de facto sieben umliegende Flüsse. In Hom. Il. XII 20-22 findet sich ein Katalog der Flüsse, der seltsamerweise einen Fluss mit dem Namen Heptaporos, der „Sieben-

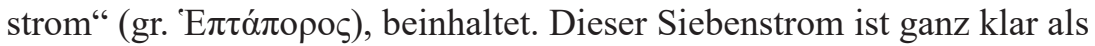

\footnotetext{
$41 \quad$ Singor 1992: 402.

42 Singor 1992: 409.

43 Wie in der Standartversion des Mythos von Nergal und Ereškigal angegeben, vgl. Dalley, 2000: 170.

44 Ehrlich 1908: 637-638.
} 
gewaltige Strömung, die aus allen sieben umliegenden Flüssen zusammen entstanden sein soll, zu verstehen.

\section{NACH DER FLUT - PAŠITTU UND SPHINX}

Die mesopotamischen Flutmythen enden nicht mit der Flut oder mit dem Überleben der Helden. Sie beinhalten stets die neuen Bedingungen für den Fortbestand der Menschheit. Da der mesopotamische Schöpfungs- und Flutmythos besonders auf eine unkontrollierte demographische Entwicklung der Menschheit anspielt, ist klar, dass es dies ist, dem die Götter einen Riegel vorsetzten. Um das Geplärre der Menschen zu dämpfen, beschließen sie das demographische Wachstum zu kontrollieren. Jahwe beschränkt die Dauer der menschlichen Lebenszeit auf 120 Jahre (Gen. 1: 6, 3). Vergleichbares geschieht auch in der griechischen Mythologie - in den sog. Weltaltern der Antike (Hes. Op. 106-201). Die Akkadier beschreiben eine Art Geburtenkontrolle, für die der Dämon Pašittu zuständig ist:

\section{[a]p-pu-na ša-lu-uš-tum li-i[b]-ši i-na ni-ši \\ i-na ni-ši a-li-it-tum-ma la a-li-it-tum \\ li-ib-ši-ma i-na ni-ši pa-ši-it-tu \\ li-is-ba-at še-er-ra / i-na bi-ir-ku a-li-it-tit ${ }^{45}$}

Es gebe außerdem eine Dritte (Kategorie) unter den Leuten!

Es gebe unter den Leuten die (Frauen), die gebären und nicht gebären, Und es wandle unter den Leuten Pašittu ${ }^{46}$, die das Kind vom Schoss jener stiehlt, die es gebar! $!^{47}$

Pašittu ist ein anderer Name für Lamaštu (sum. Dimme), Schwester von Ištar. Lamaštu ist der Dämon, der Zeugungs- und Empfängnisunfähigkeit, Fehl- und Totgeburten, das Sterben im Kindbett, sowie Müttersterblichkeit verursacht. Dabei ist Lamaštu eine frühe Version der

\footnotetext{
45 Originaltext aus Atrahasis-Epos III 7, 1-4 nach Lambert, Millard 1969: 102-103.

46 Wörtl. „der Auslöscher, die Auslöscherin“, vgl. Bane 2012: 250, s.v. „Pashitttu“ zusammen mit der angegebenen Literatur.

47 Übers. M.J.; das Addendum „Kategorie“ nach der Übers. von W.G. Lambert und A.R. Millard.
} 
hebräischen Lilith und erinnert an die griechische Lamia. Auch Männer sollen sich vor ihr gefürchtet haben und von ihr keineswegs verschont worden sein. Es scheint, als sei auch im thebanischen Sintflutmythos eine solche Gestalt Teil der neuen, post-diluvischen Ordnung. Ein Passus aus Hesiod's Theogonie (Z. 326) erinnert an das obige Zitat:

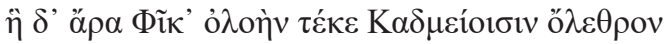

Auch die verderbliche Fix, zum Weh der Kadmeier, gebar sie ${ }^{48}$

Aus dem verlorenen Ödipus-Epos hat zudem das folgende Fragment erhalten:

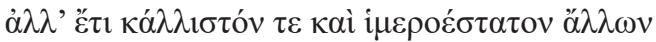

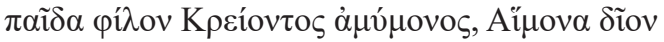

Und noch das hübscheste und liebenswürdigste von allen,

Das liebe Kind des edlen Kreon, den strahlenden Haemon ${ }^{49}$ [raubte die Sphinx]

Hinzu kommt die Erläuterung Apollodoros', dass Hera die Sphinx gesandt habe (Apoll. III 5, 8). Erich Bethe, der Heras Beziehung zu Laios für essentiell hielt, beschreibt auch beiläufig, die Sphinx ,habe Groß und Klein geraubt ${ }^{\text {" } 50}$. Dem ist unter anderem deswegen kaum zu widersprechen, weil das Rätsel der Sphinx eine Anspielung auf den Geburtsakt aufweist. Obwohl uns erst durch Athenaios (X 83) und Apollodor (III 5, 8) das Rätsel verhältnismäßig spät überliefert wird, haben wir durch eine Vase aus der ersten Hälfte des 5. Jh. v. Chr. Gewissheit: Es war bereits zu jener Zeit weit verbreitet und bekannt ${ }^{51}$. Erneut sei es hier in der Apollodor'schen Kurzfassung vorgetragen:

Übers. nach J.H. Voß.

Übers. M.J.

Bethe 1891: 13-17.

Goldman 1911: 378-385. 


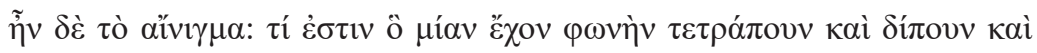

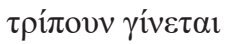

Es lautete aber das Rätsel: Was ist das, das eine einzige Bezeichnung hat und vierfüßig und zweifüßig und dreifüßig wird? ${ }^{52}$

Das Verb gr. yífvouaı wird auch in Ath. X 83 vorgefunden. Es ist

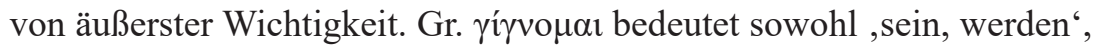
als auch ,geboren werden" - Letzteres besonders im Kontext von Menschen, so wie eben auch im Falle des Rätsels der Sphinx. Die konjugier-

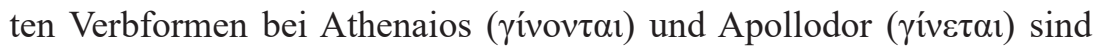
zwar grammatikalisch unterschiedlicher Numeri, aber stets im Präsens des Indikativs. Sie können also problemlos als Verben mit Futur-Bedeutung gelesen werden. Fortan beziehe sich die Sphinx in ihrem Rätsel auf den Empfang, die Geburt des Wesens, über das sie spricht. Gesandt von Hera, der Ehegöttin, die - wie Bethe bewiesen zu haben scheint - wegen Laios' Ehebruch an Jokasta durch den Raub des Chrysippos unversöhnt

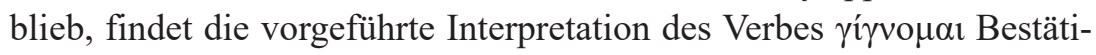
gung. Hera übernimmt damit die Rolle der Muttergöttin Nintu aus dem Atrahasis-Epos, das die menschliche Geburt ohnehin in diesem Mythos in der Fassung des Verfassers Ku-Aya besonders stark hervorhebt ${ }^{53}$. Im Lichte dessen zeichnet sich ein Bild der Sphinx, das eine verblüffende und kaum abzuweisende Ähnlichkeit mit dem der Pašittu aufweist.

Die Sphinx ist uns zwar aus der Ödipussage bekannt, doch ist sie dort mit Gewissheit sekundär ${ }^{54}$. Abgeleitet von gr. $\sigma \varphi \hat{i} \gamma \gamma \omega$ (dt. würgen) ist der Name Sphinx eine Lehnübersetzung aus einer semitischen Sprache, in der Lamaštu auch unter dem Namen „die Würgerin“ (phön. hnqqt)

\footnotetext{
52 Übers. nach P. Dräger.

53 Hera ist auch die Ursache einer Verbannung der Giganten in den Tartatos (Hom. Od. VII 58-60), die Noegel (1998: 415) mit Ogyges und Og, sowie göttlicher Bestrafung verbindet.

$54 \quad$ Edmunds 1984: 147-173.

55 Die Vokabel ḥnqt taucht in einem Text auf einem viel diskutierten Amulett aus Arslan Tasch auf. Eine gute Bearbeitung des Stoffes findet sich in dem unveröffentlichten Artikel von Chuck Häberl (dem ich für das Besprechen des Textes mit mir zu herzlichstem Dank verpflichtet bin), siehe Literaturverzeichnis unter: Häberl.
} 
bekannt gewesen ist ${ }^{56}$. Auch in akkadischen Texten finden sich zahlreiche Anspielungen auf den Würgegriff der Lamaštu (vgl. z.B. Lam. I Inc. 3 (OB1) oder Lam. I Inc. 5) ${ }^{57}$. Das Würgen der beiden Dämonen - Sphinx und Lamaštu - ist mit dem Glauben an eine Art direkten Kanal von den weiblichen Geburtsorganen bis hin zur Luftröhre zu vereinen. Zuletzt konnte Bachvarova diesen anatomischen Fehlglauben aus dem antiken Nahen Osten bis in die Ägäis verfolgen und in Mythen belegen $^{58}$. Es scheint also, dass „das Weh der Thebaner" und ihre böswillige Mordlust auf Pašittu-Lamaštu zurückzuführen ist. Wenn dem so ist, dann stellt sich die Frage nach einem thebanischen Flutmythos, dem die Sphinx angehören könnte: Dieser wurde oben rekonstruiert. Somit erhält man auch einen Hinweis darauf, dass die Ogygische Sintflut älter

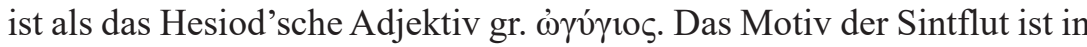
Griechenland somit deutlich früher als 550 v. Chr., in Anlehnung an die Adaption einer thebanischen Flut in der Ilias, gewiss als vor-homerisch zu datieren.

\section{FAZIT}

Aus der Erwägung der verhältnismäßig späten Überlieferungen (Paus. XI 5,1) einer thebanischen Sintflut heraus, ist es möglich, eine weitaus ältere Tradition zu erfassen. Die Homerische Epik bietet viele Möglichkeiten, die vor-homerische Epik zu erschließen. So kommen auch böotische Epen und Mythen in Betracht, wie beispielsweise Bermann zeigte. Die Ilias enthält also eine Episode, die aus einem Mythos stammte, der von der Errichtung einer siebentorigen Mauer, der menschlichen Hybris, dem Zorn der Götter und schließlich der reinigenden Sintflut handelte. Nach der Flut plagt die Menschen ein von den Göttinnen Hera-Nintu gesandter Dämon. In der Geschichte über die siebentorige Mauer Thebens ist dies die berühmte Sphinx. In ihrer Funktion und ihrem Namen erscheint sie als griechisches Gegenstück zur mesopotamischen

\footnotetext{
56 Die Etymologie von gr. $\sigma \varphi \dot{\gamma} \gamma \gamma \omega$ scheint nach wie vor korrekt zu sein, auch wenn es zwischenzeitlich sehr viele alternative Herleitungsversuche gegeben hat, dafür siehe Jagiełło 2018: 71-82.

$57 \quad$ Farber 2014: 148-149, 156-157.

58 Bachvarova 2013: 415-446.
} 
Lamaštu. Ogyges, dessen Namen die Sintflut im Laufe der Zeit annahm, mag bloß der archetypische Urahne sein, ein Vorvater derer, für die diese Sintflutsage relevant gewesen ist. (O)Gyges, der Ahne, vielleicht auch ein Wassergott, ein Okeanos, stammt wahrscheinlich aus dem hethitischkarischen Kulturkreis, wo er verehrt wurde. Sowohl die Griechen entnahmen vermutlich ihren Ogyges, als auch die Hebräer ihren Og diesem anatolischen Kult. Es bleibt jedoch offen, wie und wann genau dies geschehen ist. Ebenso bleibt ungewiss, weshalb Ogyges/Og in Flutmythen vertreten ist. Ogyges ist nämlich bereits bei Homer und Hesiod mit dem Element Wasser verbunden. Es liegt gar nahe, der verlockenden Hypothese einer Etymologie von gr. ' $\Omega \kappa \varepsilon \alpha v o ́ \varsigma$ zu erliegen. Die Insel Ogygia liegt ja bekanntermaßen auf dem Okeanos-Strom, zwischen Diesseits und Jenseits, eben wie die Heimat des Utnapischtim im GilgameschEpos, das Homer in seiner Odyssee als Inspiration diente. So auch Styx, die Hesiod'schen „ogygischen Gewässer“ - ebenfalls zwischen Diesund Jenseits. Meines Erachtens nach würde die Erklärung genügen, Ogyges/Og habe als göttlicher Urahne den Zugang in einen Flutmythos

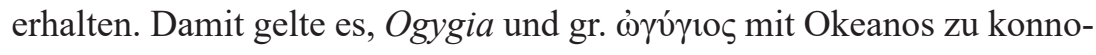
tieren lediglich als einen Interpretationsfehler unsererseits zu betrachten. Dennoch belasse ich hiermit die Frage unbeantwortet, weshalb Ogyges und $\mathrm{Og}$ in den Flutmythen auftauchen.

\section{LITERATUR}

Bachvarova M.R., 2013, 'Io and the Gorgon: Ancient Greek Medical and Mythical Constructions of the Interaction Between Women's Experiences of Sex and Birth', Arethusa 46/3, S. 415-446, https://doi.org/10.1353/are.2013.0022.

Bachvarova M.R., 2016, From Hittite to Homer: The Anatolian Background of Ancient Greek Epic, Cambridge, https://doi.org/10.1017/CBO9781139048736.

Bane T., 2012, Encyclopedia of Demons in World Religions and Cultures, Jefferson-London.

Beekes R.S.P., van Beek L., 2010, Etymological Dictionary of Greek, Leiden-Boston.

Berman D.W., 2015, Myth, Literature, and the Creation of the Topography of Thebes, Cambridge, https://doi.org/10.1017/CBO9781139924863.

Bethe E., 1891, Thebanische Heldenlieder, Leipzig. 
Brown J.P., 1995, Israel and Hellas, Berlin-New York.

Buttmann P. K., 1828/1829, 'Ueber den Mythos der Sündflut', [in:] Mythologus, Bd. 1, Berlin.

Cadwell R.S. (Übers., Hrsg.), 1987, Hesiod, Theogony, Cambridge.

Calder W.M., 1988, 'New Light on Ovid's Story of Philemon and Baucis', [in:] The Flood Myth, A. Dundes (Hrsg.), Berkeley 1988, S. 101-111.

Campbell A. (Hrsg.), 1992, Bacchylides, Corinna, Greek Lyric, IV: Bacchylides, Corinna, and Others, Cambridge.

Dalley S., 2000, Myths from Mesopotamia. Creation, the Flood, Gilgamesh and Others, Oxford.

Digital Fragmenta Historicorum Graecorum [DFHG], Acusilaus, http://www.dfhg-project.org/DFHG/digger.php?what\%5B\%5D=author\%7CACUSILAUS\&onoffswitch $=$ on [Zugriff am: 19.10.2019].

Digital Fragmenta Historicorum Graecorum [DFHG], Hellanicus, http://www. dfhg-project.org/DFHG/digger.php?what $\% 5 \mathrm{~B} \% 5 \mathrm{D}=$ =author\%7CHELLANICUS\&onoffswitch $=$ on [Zugriff am: 19.10.2019].

Edmunds L., 1984, 'The Sphinx in the Oedipus Legend', [in:] Oedipus. A Folklore Casebook, L. Edmunds, A. Dundes (Hrsg.), New York, S. 147-173.

Ehrlich H., 1908, 'König Ogygos', Rheinisches Museum für Philologie 63, S. 636-639.

Falls T.B. (Übers.), 2008, Saint Justin Martyr, First Apology, the Second Apology, Dialogue with Trypho, Exhortation to the Greeks, Discourse to the Greeks, the Monarchy of the Rule of God, Washington D.C. [Reprint].

Farber W., 2014, Lamaštu. An Edition of the Canonical Series of Lamaštu Incantations and Rituals and Related Texts from the Second and First Millenia B.C., Winona Lake.

Fontenrose J.E., 1974, Python: A Study of Delphic Myth and Its Origins, New York.

Fowler R.L., 2007, Early Greek Mythography I: Texts, Oxford-New York.

Fowler R.L., 2013, Early Greek Mythography II: Commentary, Oxford.

Friedländer G. (Übers.), 1916, Pirkei De-Rabbi Eliezer XXIII, London.

Goldman H., 1911, 'Two Unpublished Oedipus Vases in the Boston Museum of Fine Arts', American Journal of Archaeology 15, S. 378-385, https://doi. org/10.2307/497415.

Häberl Ch.G., Arslan Tash Amulet No. 1 (AT1), https://www.academia.edu/5074216/ Arslan_Tash_Amulet_No._1_AT1_[Zugriff am: 19.10.2019]. 
Jagiełło M., 2018, 'Zur Herkunft des griechischen Wortes $\Sigma \Phi І \Gamma \Xi ’$, Classica Cracoviensia 21, S. 71-82, https://doi.org/10.12797/CC.21.2018.21.04.

Kozłowski J.M., 2018, 'Gilgamesh's Quest for Immortality (Gilg. IX-XI) as a Narratological Pattern for Odysseus's Nostos (Od. V,1-XIII,187)', Eirene. Studia Graeca et Latina 54, S. 11-31.

Lambert W.G., Millard A.R., 1969, Atra-hasis. The Babylonian Story of the Flood, Oxford.

Latte K., Hansen P.A., Cunningham I.C. (Hrsg.), 2009, Hesychius Alexandrinus, Hesychii Alexandrini Lexicon, Bd. IV: T- $\Omega$, Berlin-New York, https://doi. org/10.1515/9783110222104.

Matthews V.J., 1976, Panyassis of Halikarnassos: Text and Commentary, Leiden.

Niesiołowski-Spanò Ł., 2015, Goliath's Legacy. Philistines and Hebrews in Biblical Times, Wiesbaden, https://doi.org/10.2307/j.ctvc2rn1w.

Noegel S.B., 1998, 'The Aegean Ogygos of Boeotia and the Biblical Og of Bashan: Reflections of the Same Myth', Zeitschrift für die alttestamentliche Wissenschaft 110, S. 411-426, https://doi.org/10.1515/zatw.1998.110.3.411.

Roberto U., 2011, 'From Hellenistic to Christian Universal History. Julius Africanus and the Atthidographers on the Origins of Athens', Zeitschrift für Antikes Christentum 14/3, S. 525-539, https://doi.org/10.1515/zac.2010.28.

Rölling W., 1974, 'Eine neue phönizische Inschrift aus Byblos', Neue Ephemeris für Semitische Epigraphik 2, S. 1-15.

Schmidt M. (Hrsg.), 1867, Hesychius Alexandrinus, Hesychii Alexandrini Lexicon, Jenae.

Singor H.W., 1992, 'The Achaean Wall and the Seven Gates of Thebes', Hermes 120/4, S. 401-411.

Sowa C.A., 1984, Traditional Themes and the Homeric Hymns, Chicago.

Staab G., 2006, 'Chronographie als Philosophie. Die Urwahrheit der mosaischen Überlieferungen nach dem Begründungsmodell des Mittelplatonismus bei Julius Africanus (Edition und Kommentierung von Afric. Chron. fr. 1)', [in:] Julius Africanus und die christliche Weltchronik, M. Wallraff (Hrsg.), BerlinNew York, S. 61-82.

Takmer B., Akdodu-Arca E.N., 2001/2002, 'Comments on the Fragment by Panyasis Based on the Personage of Ogyges: A Re-Evaluation of the Myth of „Bellerophon“ and the Early History of Lycia', Adana 5, S. 1-34),

http://www.akmedadalya.com/ozet_en.php?catagoryID=5\&articleID $=72$ [Zugriff am: 19.10.2019] 
Webster N., 1810, 'Origin of Mythology', [in:] Memoirs of the Connecticut Academy of Arts \& Sciences, Bd. 1, New Haven, S. 175-216.

West M.L., 1997, The East Face of Helicon, Oxford.

West M.L., 1966, Hesiod's Theogony, Oxford.

Wissowa G., 1937, Paulys Realenzyklopädie der classischen Altertumwissenschaft, Bd. 17, Stuttgart.

Wyatt N., 2006, Religious Texts from Ugarit, London. 